\title{
HERZLICH WILLKOMMEN
}

Liebe Freundinnen und Freunde des Lebku-

chenkongresses,

wir freuen uns sehr, Sie zum 48. Fortbildungskongress für Assistenzpersonal in der Radiologie, Nuklearmedizin und Strahlentherapie einladen zu dürfen!

In diesem Jahr wollen wir uns dem neuen Strahlenschutzrecht widmen. Gemeinsam mit Ihnen möchten wir die ersten Erfahrungen austauschen und bereits spürbare Veränderungen in der Radiologie diskutieren, die uns auch noch in den kommenden Jahren beschäftigen werden. Auch in den einzelnen Fachdisziplinen widmen wir uns hochrelevanten Themen, beispielsweise dreht sich in der Röntgendiagnostik alles um die Wirbelsäule. Nuklearmedizinisch Interessierte können mehr zu posttherapeutischer Bildgebung, modernen Therapieverfahren und den Strahlenschutz lernen. Und in die Strahlentherapiesitzungen gestalten wir ganz nach dem Motto „präzise und interdisziplinär“.

Mit dem Lebkuchenkongress haben alle Teilnehmerinnen und Teilnehmer außerdem die Gelegenheit, direkt ihre Fachkunden und Kenntnisse zu aktualisieren, seien es Röntgendiagnostik, Nuklearmedizin oder Strahlentherapie. Auch interessierte Ärztinnen und Ärzte können diese Chance gerne nutzen.

Einmal mehr wird uns die elegante und denkmalgeschützte Meistersingerhalle in Nürnberg den passenden Rahmen für unseren Kongress bieten. Zentral im Herzen der Stadt gelegen, ist sie optimal mit öffentlichen Verkehrsmitteln erreichbar. Auch Parkplätze stehen ausreichend zur Verfügung, und viele Hotels sind einfach zu Fuß erreichbar. Und was könnte schöner sein, als nach einem lehrreichen Tag mit viel kollegialem Austausch entspannt über den festlich geschmückten Christkindlesmarkt zu spazieren und Glühwein und Lebkuchen zu genießen.

Wir freuen uns auf Sie!

Katja Röhr

Prof. Dr. Dr. R. Loose

Dr. rer. nat. M. Wucherer 